\title{
Strategies to Reduce Mechanical Ventilation and Bronchopulmonary Dysplasia in Preterm Infants
}

Bronchopulmonary dysplasia (BPD) continues to be one of the common complications of premature birth. The paper by Freidman et al in this issue of Respiratory CARE shows the effectiveness of establishing a balanced approach to reduce the exposure to mechanical ventilation, with the ultimate goal of reducing the incidence of BPD in premature infants of very low birth weight. ${ }^{1}$

\section{See the Original Study on Page 1134}

As evidenced by the recent trials, a large proportion of infants can be adequately managed with CPAP, with comparable rates of BPD to those achieved in mechanically ventilated infants. ${ }^{2,3}$ These trials also made clear that a significant proportion of infants initially started on CPAP eventually require mechanical ventilation, which was more evident among infants of lower gestational age. In the cohort reported by Friedman et al, $60 \%$ of VLBW infants required ventilation on day 1 , and more than $30 \%$ on day 7 . Hence, once the infants are intubated and placed on mechanical ventilation, the respiratory care strategy becomes increasingly relevant.

In this cohort the proportion of extubation failure in the first era, when ventilator driven CPAP was used, was similar to that in the second era, when bubble CPAP was used after extubation. This is in agreement with existing data from clinical trials that showed none of the CPAP delivery methods offers advantages over the rest in reducing extubation failure. ${ }^{4,5}$

To manage ventilated infants, Friedman et al established a combined approach of surfactant administration with immediate extubation (the INSURE strategy) as well as a weaning protocol for rapid extubation to bubble CPAP,

The authors have disclosed no conflicts of interest.

Correspondence: Nelson Claure MSc PhD, Division of Neonatology, Department of Pediatrics, PO Box 016960, R-131, University of Miami, Miami FL 33101. E-mail: nclaure@miami.edu.

DOI: $10.4187 /$ respcare. 02595 and a reintubation protocol for those infants failing extubation. In addition, the authors report an increased use of vitamin A, and reduced rates of ductal ligation in the second era. The authors demonstrate the association between this combined approach and the observed reduction in the duration of mechanical ventilation and the incidence of BPD.

Although this study has the known limitations of a retrospective data analysis, it underlines the fact that prevention of BPD requires a multifaceted strategy. This study also reminds us that, following the institution of strategies to improve one outcome, it is prudent to monitor for the occurrence of unexpected increases in morbidities or mortality.

Nelson Claure MSc PhD
Jose Antonio Bello MD
Deepak Jain MD
Division of Neonatology
Department of Pediatrics
University of Miami Miller School of Medicine
Miami, Florida

\section{REFERENCES}

1. Friedman CA, Menchaca RC, Baker MC, Rivas CK, Laberge RN, Rios EH, et al. Bubble nasal CPAP, early surfactant treatment, and rapid extubation are associated with decreased incidence of bronchopulmonary dysplasia in very-low-birth-weight newborns: efficacy and safety considerations. Respir Care 2012;58(7):1134-1142.

2. Finer NN, Carlo WA, Walsh MC, Rich W, Gantz MG, Laptook AR, Yoder BA, et al. Early CPAP versus surfactant in extremely preterm infants. N Engl J Med 2010;27;362(21):1970-1979.

3. Morley CJ, Davis PG, Doyle LW, Brion LP, Hascoet JM, Carlin JB; COIN Trial Investigators. Nasal CPAP or intubation at birth for very preterm infants. N Engl J Med 2008;14;358(7):700-708.

4. Gupta S, Sinha SK, Tin W, Donn SM. A randomized controlled trial of post-extubation bubble continuous positive airway pressure versus Infant Flow Driver continuous positive airway pressure in preterm infants with respiratory distress syndrome. J Pediatr 2009;154(5): 645-650.

5. Stefanescu BM, Murphy WP, Hansell BJ, Fuloria M, Morgan TM, Aschner JL. A randomized, controlled trial comparing two different continuous positive airway pressure systems for the successful extubation of extremely low birth weight infants. Pediatrics 2003.; 112(5):1031-1038. 